\title{
MAN.09 - Regulatory Processes and Standards Required for Marketing Authorization of Vaccines in Brazil
}

\author{
Sheila Barros Matsuoka ${ }^{1 *}$; Laura Gomes Castanheira ${ }^{2}$; Ivna Alana da Silveira ${ }^{1}$. \\ 1Fiocruz/Bio-Manguinhos; \\ 2Without bond Institutional, with personal interest in the area.
}

Introduction: The development of a vaccine is a complex process that requires several steps, starting with identification of new targets, technological development, pre-clinical studies, clinical trials, submission of the registration process to end in the marketing authorization. Brazilian health standards are a complex of specifications to be fulfilled and dispersed by various regulations, which make it difficult to obtain the registration with ANVISA. Due to the lack of specific guidelines and considering that ANVISA can make use of guidelines and recommendations of International Regulatory Agencies or Health Organizations, since they have a cooperation partnership, it is important to elaborate a Specific Guidance to be follow by vaccines manufacturers and registry holders. This Guidance describes the standards adopted by ANVISA, FDA, EMA, ICH and WHO, related to studies of efficacy, safety and quality that vaccines must meet in order to obtain marketing authorization.

Objective: The objective of this work was to elaborate a Specific Guidance, with details on the evidences that must be obtained to demonstrate vaccine efficacy, safety and quality in order to obtain ANVISA's marketing authorization.

Methodology: A detailed investigation of a diverse worldwide health legislation (ANVISA, FDA, EMA Regulatory Agencies, WHO and ICH) related to technological development, clinical and vaccine registration was carried out as described below:

a) Legislation available on the ANVISA website composed by 62 documents;

b) Legislation available on FDA, EMA, WHO and ICH websites composed by 120 documents.

Results: After prospection, all information was compiled to make the Guidance. In addition to all the information described in the Guidance, two instructional annexes models were made. The first is related to pre-clinical and clinical studies and the second to the product registration processes. These annexes were created like Specific Forms to be used as a working tool to meet regulatory standards demanded by ANVISA and other Regulatory Agencies for technological development, clinical trials and vaccines registration. It will be very useful to researchers and project managers that work on the vaccine development.

Conclusion: The work will allow interested stakeholders in the area of technological development, clinical research and vaccine registration to strengthen knowledge regarding applicable regulatory processes. It is also intended to contribute to the discussion on the improvement of national regulatory system, using a comparative analysis of mandatory compliance procedures in important International Regulatory Agencies, identifying the main differences and similarities. The Guidance will contribute to the management of the regulatory processes in vaccine development in Brazil.

Keywords: Vaccines; Regulatory requirements; Marketing Authorization 